\title{
SOME REMARKS ON THE INVARIANT SUBSPACE PROBLEM FOR HYPONORMAL OPERATORS
}

\author{
VASILE LAURIC
}

(Received 11 February 2001)

\begin{abstract}
We make some remarks concerning the invariant subspace problem for hyponormal operators. In particular, we bring together various hypotheses that must hold for a hyponormal operator without nontrivial invariant subspaces, and we discuss the existence of such operators.
\end{abstract}

2000 Mathematics Subject Classification. 47B20, 47A15.

Let $\mathscr{H}$ be a separable, infinite-dimensional, complex Hilbert space and denote by $\mathscr{L}(\mathscr{H})$ the algebra of all linear and bounded operators on $\mathscr{H}$. An operator $T \in \mathscr{L}(\mathscr{H})$ is called hyponormal (notation: $T \in H(\mathscr{H})$ ) if $\left[T^{*}, T\right]:=T^{*} T-T T^{*} \geq 0$, or equivalently, if $\left\|T^{*} x\right\| \leq\|T x\|$ for every $x \in \mathscr{H}$.

The purpose of this paper is to use several results that may be applied to the invariant subspace problem (ISP) for hyponormal operators and thus to bring into focus what remains to be done to solve the problem completely. We begin by recalling some standard notation and terminology to be used. For a (nonempty) compact subset $K \subset \mathbb{C}$, we denote by $C(K)$ the Banach algebra of all continuous complex-valued functions on $K$ with the supremum norm, by $\operatorname{Rat}(K)$ the subalgebra of $C(K)$ consisting of all rational functions with poles off the set $K$, and by $R(K)$ the closure in $C(K)$ of $\operatorname{Rat}(K)$. For $T \in \mathscr{L}(\mathscr{H})$, the spectrum of $T$ is denoted by $\sigma(T)$ and the algebra $\{r(T)$ : $r \in \operatorname{Rat}(\sigma(T))\}$ by $\operatorname{Rat}(T)$. The rational cyclic multiplicity of $T$ (notation: $m(T)$ ) is the smallest cardinal number $m$ with the property that there are $m$ vectors $\left\{x_{i}\right\}_{0 \leq i<m}$ in $\mathscr{H}$ such that $\vee\left\{A x_{i} \mid 0 \leq i<m, A \in \operatorname{Rat}(T)\right\}=\mathscr{H}$. For a bounded (nonempty) open subset $U \subset \mathbb{C}$, one denotes by $\mathbf{H}^{\infty}(U)$ the Banach algebra of those analytic complex-valued functions on $U$ with the property that $\|f\|_{\infty, U}:=\sup _{z \in U}|f(z)|<\infty$. The ideal of all compact operators on $\mathscr{H}$ will be denoted by $\mathbb{K}=\mathbb{K}(\mathscr{H})$. Since $\mathbb{K}$ is a two-sided, normclosed ideal in $\mathscr{L}(\mathscr{H})$, the quotient algebra $\mathscr{L}(\mathscr{H}) / \mathbb{K}$ is a $C^{*}$-algebra, which is called the Calkin algebra, and the quotient map from $\mathscr{L}(\mathscr{H})$ to $\mathscr{L}(\mathscr{H}) / \mathbb{K}$ will be denoted by $\pi$. For $T$ in $\mathscr{L}(\mathscr{H})$, we write $\sigma_{e}(T)$ (resp., $\sigma_{\text {re }}(T), \sigma_{\text {le }}(T)$ ) for the essential (resp., right-, left-essential) spectrum of $T$ (i.e., the spectrum (resp., right, left spectrum) of $\pi(T)$ ). An operator $A \in \mathbb{K}(\mathscr{H})$ is called a trace-class operator (notation: $A \in \mathscr{C}_{1}(\mathscr{H})$ ) if the series $\operatorname{tr}(|A|):=\sum_{i \in \mathbb{N}}\left\langle|A| e_{i}, e_{i}\right\rangle$ is convergent, where $|A|=\left(A^{*} A\right)^{1 / 2}$ and $\left\{e_{i}\right\}_{i \in \mathbb{N}}$ is an orthonormal basis of $\mathscr{H}$. An operator $A \in \mathbb{K}(\mathscr{H})$ is Hilbert-Schmidt operator (notation: $A \in \mathscr{C}_{2}(\mathscr{H})$ ) if $A^{*} A \in \mathscr{C}_{1}(\mathscr{H})$. For a selfadjoint operator $A \in \mathscr{L}(\mathscr{H}), A_{-}$will denote its negative part $(|A|-A) / 2$. Finally, $\mu$ will denote planar Lebesgue measure defined on the Borel subsets of $\mathbb{R}^{2}$. 
A beautiful generalization of the Berger-Shaw inequality for hyponormal operators [3] was given by Voiculescu.

THEOREM 1 (see [17]). If $\left[T^{*}, T\right]_{-} \in \mathscr{C}_{1}(\mathscr{H})$ and $X \in \mathscr{C}_{2}(\mathscr{H})$ is such that $m(T+X)<$ $+\infty$, then $\left[T^{*}, T\right] \in \mathscr{C}_{1}(\mathscr{H})$ and $\pi \operatorname{tr}\left(\left[T^{*}, T\right]\right) \leq m(T+X) \mu(\sigma(T+X))$.

For purposes of finding a nontrivial invariant subspace (n.i.s.) for an arbitrary operator $T \in \mathscr{L}(\mathscr{H})$, one may assume that every nonzero vector in $\mathscr{H}$ is cyclic for $T$, and hence that $m(T)=1$. Thus the following corollary (of Theorem 1 or the earlier Berger-Shaw inequality) is useful.

COROLLARY 2. Every hyponormal operator $T$ in $\mathscr{L}(\mathscr{H})$ with a rational cyclic vector (i.e., $m(T)=1$ ) is essentially normal (i.e., $\pi(T)$ is normal in the Calkin algebra).

When looking for a n.i.s. for an arbitrary $T$ in $\mathscr{L}(\mathscr{H})$, one knows (cf. [10]) from a deep theorem of Apostol, Foiaş, and Voiculescu [2] that $T$ may be assumed to belong to the class $\mathscr{B} 2 \mathcal{T}$ ( $\mathscr{H}$ ) of biquasitriangular operators, (see [10] for the definition and a characterization). If we denote by $\mathscr{E} \mathcal{N}(\mathscr{H})$ the collection of essentially normal operators on $\mathscr{H}$ and by $(N+K)(\mathscr{H})$ the collection of those operators in $\mathscr{L}(\mathscr{H})$ which can be written as a sum of a normal and a compact operator, then a deep result of Brown-DouglasFillmore can also be applied.

THEOREM 3 (see [5]). The equality $\mathscr{B} \mathscr{T} \mathcal{T}(\mathscr{H}) \cap \mathscr{E} \mathcal{N}(\mathscr{H})=(N+K)(\mathscr{H})$ holds.

It is thus immediate from Theorems 1 and 3 and Corollary 2 that if there exists $T$ in $H(\mathscr{H})$ without a n.i.s., then $\left[T^{*}, T\right] \in \mathscr{C}_{1}(\mathscr{H})$ and $T \in(N+K)(\mathscr{H})$.

A hyponormal operator is called pure if it does not have a nonzero reducing subspace to which its restriction is a normal operator. Obviously an operator in $H(\mathscr{H})$ without a n.i.s. is pure. The following result of Putnam [14] leads to another reduction of the ISP for hyponormal operators.

THEOREM 4. Let $T \in \mathscr{L}(\mathscr{H})$ be a pure hyponormal operator. If $\Delta \subset \mathbb{C}$ is an open set, then $\mu(\Delta \cap \sigma(T))>0$ whenever $\Delta \cap \sigma(T) \neq \varnothing$.

This says that each point of the spectrum of such a $T$ has positive planar density, and thus we may assume of a hyponormal operator $T$ without a n.i.s. that $\sigma(T)$ has not only positive $\mu$-measure but positive planar density at each point.

Let $A \in \mathscr{L}(\mathscr{H})$ be a selfadjoint operator and denote by $E$ the spectral measure of the operator $A$. To every vector $x \in \mathscr{H}$ one may associate the Borel measure $v_{x}$ on $\mathbb{R}$ defined by $v_{x}(\Omega)=\langle E(\Omega) x, x\rangle$ for every Borel set $\Omega \subset \mathbb{R}$. The vector $x$ is called absolutely continuous with respect to $A$ if the measure $v_{x}$ is absolutely continuous with respect to Lebesgue measure on $\mathbb{R}$. The selfadjoint operator $A$ is called absolutely continuous if every vector of $\mathscr{H}$ is absolutely continuous with respect to $A$. The following result can be found in [13], (see also [9, page 135]).

Proposition 5. If $T=X+i Y \in \mathscr{L}(\mathscr{H})$ is the Cartesian decomposition of a pure hyponormal operator, then $X$ and $Y$ are both absolutely continuous operators.

Next, recall that a subset $\Delta$ of a nonempty open set $U$ in $\mathbb{C}$ is called dominating for $U$ if $\|f\|_{\infty, U}=\sup _{\lambda \in \Delta}|f(\lambda)|, f \in \mathbf{H}^{\infty}(U)$. 
The deep invariant subspace theorem for hyponormal operators obtained by Brown in [6] on the basis of the beautiful structure theorem for such operators by Putinar [12] is the following.

THEOREM 6. Let $T \in \mathscr{L}(\mathscr{H})$ be a hyponormal operator. If there is a nonempty open set $U \subset \mathbb{C}$ such that $\sigma(T) \cap U$ is dominating for $U$, then $T$ has a n.i.s.

Since one knows (cf. [1] or [6]) that if $K$ is a (nonempty) compact set in $\mathbb{C}$ such that $R(K) \neq C(K)$, then $K$ is dominating on some nonempty open set, one gets immediately the following corollary.

COROLLARY 7 (see [6]). Any hyponormal operator $T \in \mathscr{L}(\mathscr{H})$ with $R(\sigma(T)) \neq C(\sigma(T))$ has a n.i.s.

Thus if there exist hyponormal operators $T$ without a n.i.s., then as noted above, $T \in$ $(N+K)(\mathscr{H})$ and $\sigma(T)$ must satisfy $R(\sigma(T))=C(\sigma(T))$. Moreover, it is a consequence of elementary Fredholm theory (cf. [10]) that if $T \in \mathscr{L}(\mathscr{H})$ and $\sigma_{\mathrm{le}}(T) \neq \sigma(T)$, then $T^{*}$ has point spectrum and thus $T$ has a n.i.s. Hence when looking for invariant subspaces for an arbitrary operator $T$ we may always suppose that $\sigma_{\mathrm{le}}(T)=\sigma_{\mathrm{re}}(T)=\sigma(T)$. This allows one to apply a result of Stampfli [16] to the problem.

TheOrem 8. Suppose $T \in \mathscr{E} \mathcal{N}(\mathscr{H})$ is such that $\sigma(T)=\sigma_{\mathrm{le}}(T)$ and the Calkin map $\pi: \operatorname{Rat}(T) \rightarrow \mathscr{L}(\mathscr{H}) / \mathbb{K}$ is bounded below. Then $T$ has a n.i.s.

Proof. By hypothesis, there exists a constant $M>0$ such that $\|\pi(r(T))\|_{e} \geq$ $M\|r(T)\|$ for every $r \in \operatorname{Rat}(\sigma(T))$, where $\|\cdot\|_{e}$ is the norm in the Calkin algebra. On the other hand,

$$
\|\pi(r(T))\|_{e}=\|r(\pi(T))\|_{e}=\sup _{z \in \sigma_{e}(T)}|r(z)|=\sup _{z \in \sigma(T)}|r(z)|
$$

Thus $\|r(T)\| \leq(1 / M)\|r\|_{\sigma(T)}, r \in \operatorname{Rat}(\sigma(T))$, so $\sigma(T)$ is a $(1 / M)$-spectral set for $T$. The result now follows from [16].

COROLlary 9. If $T \in H(\mathscr{H})$ and $T$ has no n.i.s., then there exist sequences $\left\{r_{n}(T)\right\}_{n \in \mathbb{N}}$ in the algebra $\operatorname{Rat}(T)$ and $\left\{K_{n}\right\}_{n \in \mathbb{N}}$ in $\mathbb{K}$ such that $\left\|r_{n}(T)\right\|=1, n \in \mathbb{N}$, and $\| r_{n}(T)-$ $K_{n} \| \rightarrow 0$. Moreover, any such sequence $\left\{r_{n}(T)\right\}_{n \in \mathbb{N}}$ has no subnet converging in the weak operator topology (WOT) to a nonzero operator.

Proof. Since $T \in H(\mathscr{H})$ has no n.i.s., $m(T)=1$, and according to Corollary 2, $T \in \mathscr{E} \mathcal{N}(\mathscr{H})$. According to Theorem $8, \pi: \operatorname{Rat}(T) \rightarrow \mathscr{L}(\mathscr{H}) / \mathbb{K}$ must not be bounded below. Thus, there are sequences $\left\{r_{n}(T)\right\}_{n \in \mathbb{N}}$ in the algebra $\operatorname{Rat}(T)$ and $\left\{K_{n}\right\}_{n \in \mathbb{N}}$ in $\mathbb{K}$ such that $\left\|r_{n}(T)\right\|=1, n \in \mathbb{N}$, and $\left\|r_{n}(T)-K_{n}\right\| \rightarrow 0$. Moreover, if there is a subnet $\left\{r_{n_{k}}(T)\right\}_{k \in \mathbb{N}}$ converging in the WOT to a nonzero operator, then $T$ has a nontrivial hyperinvariant subspace according to [8].

The following proposition simply summarizes the results mentioned above.

Proposition 10. If there exists $T \in H(\mathscr{H})$ such that $T$ has no n.i.s., then $T$ has the following properties:

(a) $\sigma_{\mathrm{le}}(T)=\sigma_{\mathrm{re}}(T)=\sigma(T)$, 
(b) $\sigma(T)$ is a connected and perfect subset of $\mathbb{C}$ such that every point of $\sigma(T)$ has positive planar density,

(c) $R(\sigma(T))=C(\sigma(T))$, and, more generally, for every nonempty bounded open set $U \subset \mathbb{C}, U \cap \sigma(T)$ is not dominating for $U$,

(d) $T=N+K$, where $N$ is a normal operator and $K$ is compact,

(e) $T=X+i Y$, where $X$ and $Y$ are absolutely continuous selfadjoint operators,

(f) $\left[T^{*}, T\right]$ is a positive semi-definite operator in $\mathscr{C}_{1}(\mathscr{H})$ with $\operatorname{tr}\left(\left[T^{*}, T\right]\right)>0$,

(g) $m(T)=1$,

(h) there exist sequences $\left\{r_{n}(T)\right\}_{n \in \mathbb{N}} \subset \operatorname{Rat}(T)$ and $\left\{K_{n}\right\}_{n \in \mathbb{N}} \in \mathbb{K}$ such that $\left\|r_{n}(T)\right\|=1, n \in \mathbb{N}$, and $\left\|r_{n}(T)-K_{n}\right\| \rightarrow 0$. Moreover, any such sequence has no subnet converging in the WOT to a nonzero operator.

This proposition raises the interesting question:

Problem 11. Are there any operators in $H(\mathscr{H})$ satisfying (a)-(h)?

This is perhaps a difficult question, which we are unable to answer at present. Moreover, the list of necessary conditions for a hyponormal operator that has no n.i.s. is larger and, of course, not all results are included in this paper. The remainder of this paper is devoted to making some progress on this question. We first recall a result from [11].

Proposition 12. Given a nonempty compact set $K \subset \mathbb{C}$ with positive density at each point, there is an irreducible, hyponormal operator with rank one self-commutator whose spectrum is $K$.

This proposition shows that to make a start toward answering Problem 11 in the affirmative, we need to construct a compact set $K$ which has properties (b) and (c) of Proposition 10 (with $K=\sigma(T)$ ). A collection of such sets $K$ may be constructed by slight variation of the following.

EXAMPLE 13. We first specify a Cantor set $C_{\left\{\theta_{n}\right\}} \subset[0,1]$ which has positive linear Lebesgue measure and, in fact, has positive linear density at each point. For this purpose we follow the notation and terminology of [4, Example 6P] and set $\theta_{n}=1 / 3^{n}$ for each $n \in \mathbb{N}$. Note that for each $n$ the closed intervals in the collection $\mathscr{F}_{n}$ have the same length-say $l_{n}$. Let $p \in C_{\left\{\theta_{n}\right\}}$ and let $(a, b)$ be an open interval containing the point $p$. Since $C_{\left\{\theta_{n}\right\}}=\cap_{n}\left(\cup\left\{I: I \in \mathscr{F}_{n}\right\}\right)$ and $l_{n} \rightarrow 0$, there exists $n_{0}$ sufficiently large that some interval $I_{0} \in \mathscr{F}_{n}$ satisfies $p \in I_{0} \subset(a, b)$. Since $C_{\left\{\theta_{n}\right\}} \cap I_{0}$ is another Cantor set, its measure can be easily calculated to be greater than $\left[l_{n_{0}}-l_{n_{0}}\left(\sum_{k \in \mathbb{N}} 1 / 3^{n_{0}+k}\right)\right]=$ $l_{n_{0}}\left(1-1 /\left(2 \cdot 3^{n_{0}}\right)\right)>0$, and thus $C_{\left\{\theta_{n}\right\}}$ has positive density at each point $p$. Let now $z_{0}$ be the point $(1 / 2,1)$ in $\mathbb{C}$, and consider the planar set

$$
K_{1}:=\left\{t p+(1-t) z_{0} \mid 0 \leq t \leq 1, p \in C_{\left\{\theta_{n}\right\}}\right\} .
$$

We will show that $K_{1}$ has the properties (b) and (c) above. By construction, the set $K_{1}$ is arcwise connected and perfect (since $C_{\left\{\theta_{n}\right\}}$ is perfect). Next, we show that every point $q$ of $K_{1}$ has positive planar density. Let $\Delta$ be an open disc in $\mathbb{C}$ such that $q \in \Delta$. Since $K_{1}$ is perfect, there exists a point $t_{0} p_{0}+\left(1-t_{0}\right) x_{0} \in \Delta \cap K_{1}$ such that 
$0<t_{0}<1$. Clearly there exist positive real numbers $t_{1}, t_{2}$ such that $0<t_{1}<t_{0}<t_{2}<1$ and some nondegenerate interval $[a, b]$ with $p_{0} \in[a, b]$ such that the trapezoid-like figure $\Gamma:=\left\{t p+(1-t) z_{0}: t_{1} \leq t \leq t_{2}, p \in C_{\left\{\theta_{n}\right\}} \cap[a, b]\right\}$ is contained in $\Delta$. Let $\alpha>0$ be the linear measure of the set $C_{\left\{\theta_{n}\right\}} \cap[a, b]$. Then the intersection of the set $K_{1} \cap \Gamma$ with each horizontal line $y=t, t_{1} \leq t \leq t_{2}$ has (linear) measure $(1-t) \alpha$. Thus, by Fubini's theorem, the $\mu$-measure of the set $K_{1} \cap \Gamma$ is $\int_{t_{1}}^{t_{2}}(1-t) \alpha d t=\left(t_{2}-t_{1}\right)\left(1-\left(t_{1}+t_{2}\right) / 2\right) \alpha>$ 0 , and hence $K_{1}$ has property (b). To check that $K_{1}$ has property (c), the following lemma is useful.

LEMMA 14. If $U$ is any bounded open set such that $U \cap K_{1} \neq \varnothing$, then there exist a point $p_{0}$ belonging to the outer boundary of $U$, an $\varepsilon>0$, and a disc $D=\left\{z \in \mathbb{C}:\left|z-p_{0}\right|<\varepsilon\right\}$ such that $D \cap K_{1}=\varnothing$.

Proof. By construction, $[0,1] \backslash C_{\left\{\theta_{n}\right\}}=\cup_{n=1}^{\infty}\left(a_{n}, b_{n}\right)$ where $\left\{\left(a_{n}, b_{n}\right)\right\}_{n=1}^{\infty}$ is the disjoint sequence of "excluded" open intervals. Thus each open triangular domain $T_{n}=\left\{t z_{0}+(1-t) p:-\infty<t<1, p \in\left(a_{n}, b_{n}\right)\right\}$ in $\mathbb{C}$ is disjoint from $K_{1}$. Since $U \cap K_{1} \neq$ $\varnothing$ and every point of $C_{\left\{\theta_{n}\right\}}$ is a limit point of end points of arbitrarily short excluded intervals, there exists some triangular domain $T_{n_{0}}$ such that $U \cap T_{n_{0}} \neq \varnothing$, and clearly any half-line joining a point of $U \cap T_{n_{0}}$ to the ideal point $|z|=+\infty$ and lying entirely in $T_{n_{0}}$ must intersect $\partial U$ in some last point (since $\partial U$ is compact), which clearly satisfies the desired conclusions.

We next show that $K_{1}$ satisfies (c) of Proposition 10. Let $U$ be a bounded open set in $\mathbb{C}$ such that $U \cap K_{1} \neq \varnothing$ and set $C=U^{-}$. Then the outer boundary of $C$ coincides with the outer boundary of $U$, and applying Lemma 14 to $U$, we get a point $p_{0}$ of the outer boundary of $U$ and an open disc $D$ centered at $p_{0}$ with radius $\varepsilon>0$ such that $D \cap K_{1}=\varnothing$. By [7, Corollary 13.3], $p_{0}$ is a peak point of $R(C)$, that is, there exists an $f_{0} \in R(C)$ such that $f_{0}\left(p_{0}\right)=1$ and $\left|f_{0}(z)\right|<1$ for $z \in C \backslash\left\{p_{0}\right\}$. Clearly $f_{0} \in \mathbf{H}^{\infty}(U)$ and $\sup _{\lambda \in U}\left|f_{0}(\lambda)\right|=1$ (since $p_{0} \in \partial U$ ) while $\sup _{\lambda \in K_{1} \cap U}\left|f_{0}(\lambda)\right|<1$ (since $\left(K_{1} \cap U\right)^{-}$ is at positive distance from $p_{0}$ and $\left|f_{0}\right|<1$ on $\left.\left(K_{1} \cap U\right)^{-} \subset C \backslash D\right)$. Thus $K_{1} \cap U$ is not dominating for $U$, and $K_{1}$ has property (c).

Let $T_{1}$ be an irreducible hyponormal operator with rank one self-commutator whose spectrum $\sigma\left(T_{1}\right)$ is the compact set $K_{1}$ described in Example 13, and whose existence is guaranteed by Proposition 12 . Thus property (f) is also satisfied. One observes that property (a) is satisfied too. Indeed, if one assumes that there exists $\lambda_{0} \in \sigma\left(T_{1}\right) \backslash \sigma_{\mathrm{le}}\left(T_{1}\right)$, then $T_{1}-\lambda_{0}$ is semi-Fredholm operator with nonpositive index (since $T_{1} \in H(\mathscr{H})$ ). Since $T_{1}$ is pure, $\sigma_{p}\left(T_{1}\right)=\varnothing$, and thus the index is negative, which implies that $\sigma\left(T_{1}\right)$ contains a nonempty open set. Obviously this is a contradiction since $\sigma\left(T_{1}\right)=K_{1}$ has no interior, and thus $\sigma\left(T_{1}\right)=\sigma_{\mathrm{le}}\left(T_{1}\right)$. In a similar way one shows that $\sigma\left(T_{1}\right)=\sigma_{\mathrm{re}}\left(T_{1}\right)$. Moreover, $T_{1} \in \mathscr{B} 2 \mathscr{T}(\mathscr{H})$ according to theorem of Apostol, Foiaş, and Voiculescu [2] since $\sigma_{e}\left(T_{1}\right)$ contains no pseudoholes or holes associated with Fredholm index different from 0 . Thus, by Theorem 3 , the operator $T_{1}$ can be written $T_{1}=N+K$, where $N$ is a normal operator and $K$ is a compact operator. Moreover, since $T_{1}$ is pure, $T_{1}=X_{1}+i Y_{1}$, where $X_{1}$, and $Y_{1}$ are absolutely continuous selfadjoint operators according to Proposition 5.

Thus we have shown that the operator $T_{1}$ has properties (a)-(f) of Proposition 10. Whether $T_{1}$ has properties $(\mathrm{g})$ and $(\mathrm{h})$ of this proposition the author is unable to 
conclude. However, techniques and results of Stampfli [15] may be applied to obtain the following theorem.

THEOREM 15. Any hyponormal operator $T$ in $\mathscr{L}(\mathscr{H})$ such that $\sigma(T)$ is the set $K_{1}$ of Example 13 has a nontrivial hyperinvariant subspace.

Proof. Consider the operator $T-(1 / 2+i)$, which has spectrum $K_{1}-(1 / 2+i)$. Define $K_{+}:=K_{1} \cap D_{1}^{-}$, where $D_{1}:=\{z \in \mathbb{C}:|z-4|<4\}$ and $K_{-}:=K_{1} \cap D_{2}^{-}$, where $D_{2}=\{z \in \mathbb{C}:|z+4|<4\}$. Then $\sigma(T)=K_{+} \cup K_{-}, K_{+} \cap K_{-}=\{(0,0)\}$, and $\partial D_{1} \cap K_{1}=$ $\partial D_{2} \cap K_{1}=\{(0,0)\}$. Choosing $f_{1}(z)=f_{2}(z)=z^{2}$, we may follow [15, Example 1] and observe that the operators $A_{i}:=\int_{\partial D_{i}} f_{i}(z)(z-T)^{-1} d z, i=1,2$, commute with any operator $S$ with which $T$ commutes to conclude that $T$ has a nontrivial hyperinvariant subspace.

REMARK 16. We note that it is quite easy to modify the construction of Example 13 to produce compact sets satisfying properties (b) and (c) of Proposition 10 such that the techniques of [15] of "integrating through the spectrum" are no longer available to produce nontrivial invariant subspaces for the corresponding operator $T$.

\section{REFERENCES}

[1] C. Apostol, The spectral flavour of Scott Brown's techniques, J. Operator Theory 6 (1981), no. 1, 3-12. MR 83a:47005b. Zbl 479.47003.

[2] C. Apostol, C. Foiaş, and D. Voiculescu, Some results on non-quasitriangular operators. IV, Rev. Roumaine Math. Pures Appl. 18 (1973), 487-514. Zbl 264.47007.

[3] C. A. Berger and B. I. Shaw, Selfcommutators of multicyclic hyponormal operators are always trace class, Bull. Amer. Math. Soc. 79 (1973), 1193-1199. MR 51\#11168. Zbl 0283.47018.

[4] A. Brown and C. Pearcy, An Introduction to Analysis, Graduate Texts in Mathematics, vol. 154, Springer-Verlag, New York, 1995. MR 96h:00001. Zbl 820.00003.

[5] L. G. Brown, R. G. Douglas, and P. A. Fillmore, Unitary equivalence modulo the compact operators and extensions of $C^{*}$-algebras, Proceedings of a Conference on Operator Theory (Dalhousie Univ., Halifax, N.S., 1973), Lecture Notes in Math., vol. 345, Springer, Berlin, 1973, pp. 58-128. MR 52\#1378. Zbl 277.46053.

[6] S. W. Brown, Hyponormal operators with thick spectra have invariant subspaces, Ann. of Math. (2) 125 (1987), no. 1, 93-103. MR 88c:47010. Zbl 635.47020.

[7] J. B. Conway, The Theory of Subnormal Operators, Mathematical Surveys and Monographs, vol. 36, American Mathematical Society, Rhode Island, 1991. MR 92h:47026. Zbl 743.47012.

[8] V. Lomonosov, An extension of Burnside's theorem to infinite-dimensional spaces, Israel J. Math. 75 (1991), no. 2-3, 329-339. MR 93h:47007. Zbl 777.47005.

[9] M. Martin and M. Putinar, Lectures on Hyponormal Operators, Operator Theory: Advances and Applications, vol. 39, Birkhäuser Verlag, Basel, 1989. MR 91c:47041. Zbl 684.47018.

[10] C. M. Pearcy, Some Recent Developments in Operator Theory, Regional Conference Series in Mathematics, no. 36, American Mathematical Society, Rhode Island, 1978. MR 58\#7120. Zbl 444.47001.

[11] J. D. Pincus, Commutators and systems of singular integral equations. I, Acta Math. 121 (1968), 219-249. MR 39\#2026. Zbl 179.44601.

[12] M. Putinar, Hyponormal operators are subscalar, J. Operator Theory 12 (1984), no. 2, 385-395. MR 85h:47027. Zbl 573.47016.

[13] C. R. Putnam, Commutators and absolutely continuous operators, Trans. Amer. Math. Soc. 87 (1958), 513-525. MR 20\#6659. Zbl 101.33203. 
[14] _ The spectra of completely hyponormal operators, Amer. J. Math. 93 (1971), 699708. MR 43\#6757. Zbl 219.47020.

[15] J. G. Stampfli, A local spectral theory for operators. IV. Invariant subspaces, Indiana Univ. Math. J. 22 (1972), 159-167. MR 45\#5793. Zbl 254.47007.

[16] _ An extension of Scott Brown's invariant subspace theorem: $K$-spectral sets, J. Operator Theory 3 (1980), no. 1, 3-21. MR 81e:47009. Zbl 449.47004.

[17] D. Voiculescu, A note on quasitriangularity and trace-class self-commutators, Acta Sci. Math. (Szeged) 42 (1980), no. 1-2, 195-199. MR 81k:47035. Zbl 441.47022.

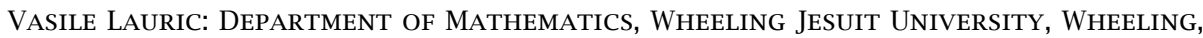
WV 26003, USA

E-mail address: 1auric@wju.edu 


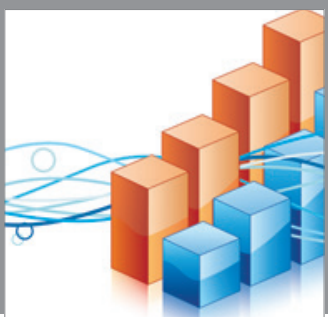

Advances in

Operations Research

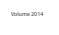

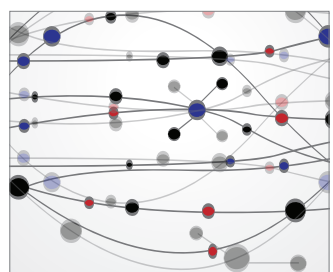

\section{The Scientific} World Journal
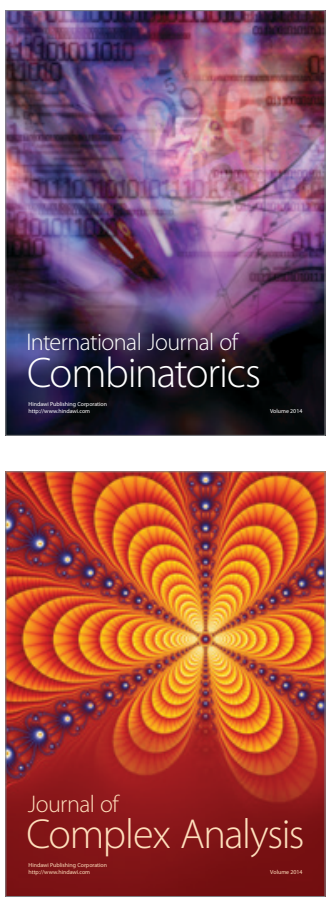

International Journal of

Mathematics and

Mathematical

Sciences
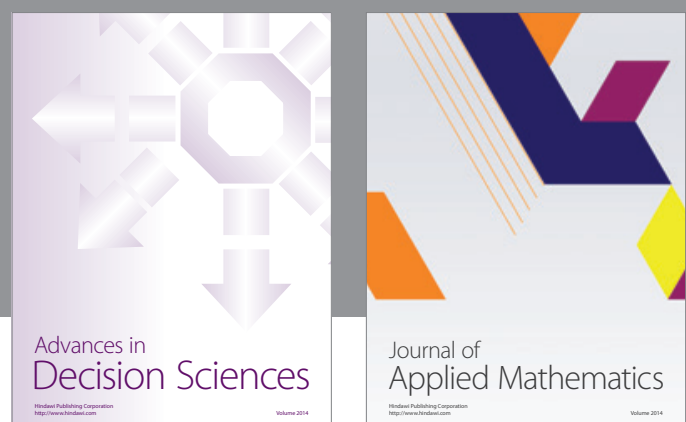

Journal of

Applied Mathematics
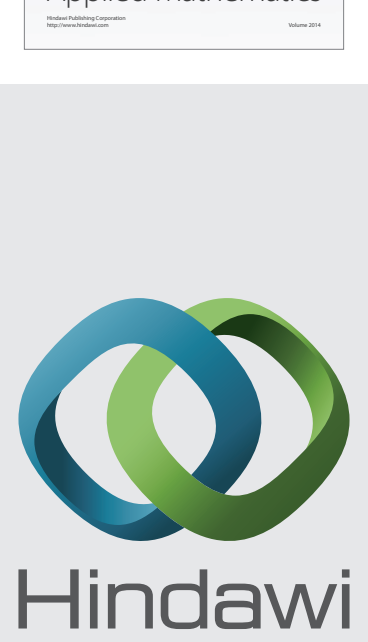

Submit your manuscripts at http://www.hindawi.com
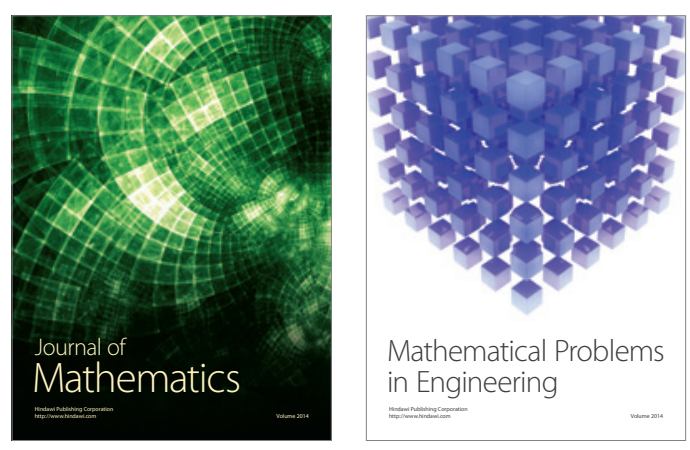

Mathematical Problems in Engineering
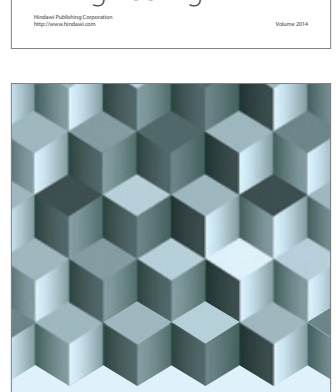

Journal of

Function Spaces
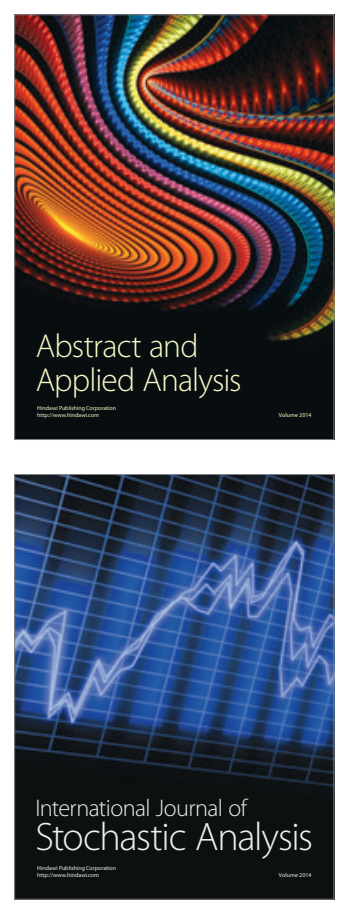

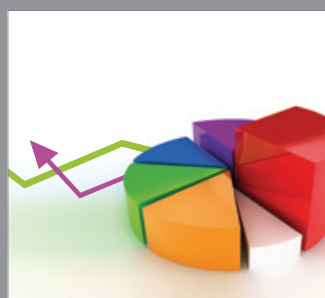

ournal of

Probability and Statistics

Promensencen
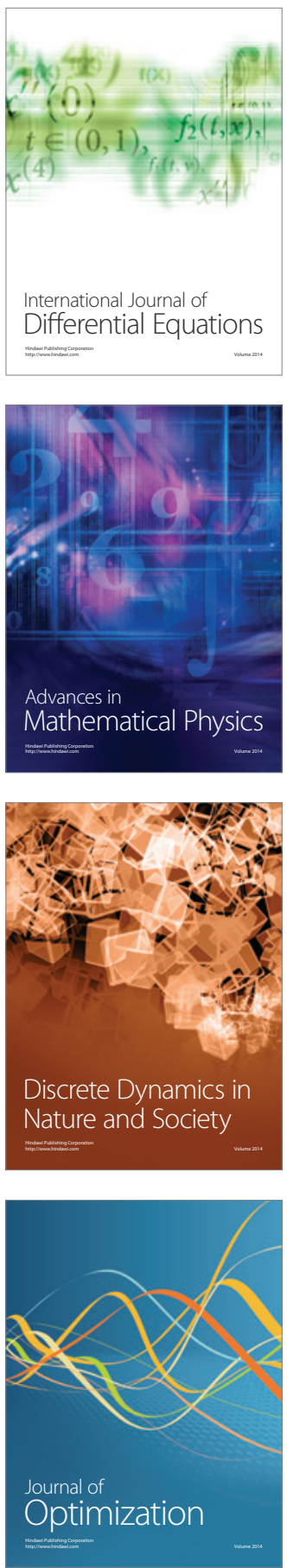